\title{
Influence of Incidence Angle on the Coherent Copolar Polarimetric Response of Rice at X-Band
}

\author{
Juan M. Lopez-Sanchez, Senior Member, IEEE, Fernando Vicente-Guijalba, \\ J. David Ballester-Berman, and Shane R. Cloude, Fellow, IEEE
}

\begin{abstract}
The coherent nature of the acquisition by TerraSAR-X of both copolar channels (HH and VV) enables the generation of many different polarimetric observables with physical interpretation, as have recently been used for monitoring rice fields. In this work, the influence of incidence angle upon these polarimetric observables is analysed by comparing three stacks of images that were acquired simultaneously at different incidence angles (22, 30 and 40 deg.) during a whole cultivation campaign. We show that the response of observables related to dominance (entropy, ratios of components) and type of scattering mechanisms (alpha angles) is not greatly influenced by incidence angle at some stages: early and advanced vegetative phase, and maturation. Moreover, the acquisition geometry drives the sensitivity to the presence of the initial stems and tillers, being detected earlier at shallower angles. This analysis is a necessary step before studying potential methodologies for combining different orbits and beams for reducing the time between acquisitions for monitoring purposes.
\end{abstract}

\section{Keywords}

Synthetic aperture radar, agriculture, polarimetry, rice

\section{INTRODUCTION}

Rice has been the subject of numerous studies aimed at monitoring with remote sensing. Among other technologies, radar is well suited for such purposes thanks to its nearly all-weather operation and day/night independence. Successful results were obtained in the past with SAR satellites operating at C- and L-band, demonstrating that mapping rice-planted areas and monitoring its evolution is feasible [1]-[5]. More recently, $\mathrm{X}$-band satellite data have been also applied to rice monitoring [6]-[8].

There exist a number of agriculture applications for which continuous observation, i.e. retrieval of information with a short refresh rate, is a key aspect. For instance, existing models devised to estimate rice production require the input of some biophysical parameters gathered at particular moments during the cultivation cycle [9], [10]. Many remote sensing activities, specially for rice cultivation in Asia, are aimed at estimating the dates of planting, heading and harvesting, which are key stages for production and management [11]. In other regions in which an integrated production is established, precision farming also demands data with fine temporal sampling to be effective (e.g. fertilisation is to be stopped at panicle initiation). Therefore, a short revisit time is mandatory to implement the required products based on images gathered by Earth observation satellites.

In the case of TerraSAR-X, revisit time with the same mode and geometry is 11 days, which is not enough for these applications. The observation rate could be increased by combining different passes (ascending and descending) and different beams (incidence angles), thus shortening the gap between successive acquisitions down to 2 or 3 days. All these acquisitions would be jointly processed under a time series approach. But before that, it is important to analyse the influence of incidence angle in the observed radar response from the rice fields. This analysis is presented here for the case of copolar polarimetry (i.e. coherent measurement of both copolar channels, $\mathrm{HH}$ and VV), as provided by TerraSAR-X in an operational mode.

The evolution of a wide number of polarimetric observables as a function of crop development was already studied for an incidence of $30 \mathrm{deg}$. in [6], [7]. These references include a detailed physical interpretation of the behaviour of different observables in terms of the phenological development of the rice fields, so it

J. M. Lopez-Sanchez, J. D. Ballester-Berman and F. Vicente-Guijalba are with the Institute for Computing Research (IUII), University of Alicante, P.O.Box 99, E-03080 Alicante, Spain (e-mail: juanma-lopez@ieee.org, fernando.vicente@ua.es, davidb@ua.es). Shane R. Cloude is with AEL Consultants, 26 Westfield Avenue, Cupar, Fife, KY15 5AA, Scotland, UK (e-mail: aelc@ mac.com). This work was supported by the Spanish Ministry of Economy and Competitiveness (MINECO) and EU FEDER, under Project TEC2011-28201-C02-02. 
will not be repeated here. Instead, this letter is focused on the relative differences and common behaviours among incidence angles. We will concentrate the analysis in justifying, from the physical point of view, the similarities and differences that we can observe in the response of these fields when we change the incidence angle. The reproducibility of our findings in other sites will depend on many different parameters (planting and cultivation practices, weather conditions, rice varieties, etc.). Hence, this study provides some guidelines about what to expect in copolar observables acquired over rice fields at X-band when changing the incidence angle between 22 and $40 \mathrm{deg}$.

In the literature a complete study for angles from 25 to $55 \mathrm{deg}$. was carried out in [12] with a scatterometer, covering the cultivation cycle of one rice field with daily measurements. Unfortunately, that study only analysed backscattering coefficients, not other polarimetric observables. Therefore, we extend here such a study to a wider observation space and to satellite imagery. We are especially interested in three types of polarimetric observables that have not been widely exploited yet by other researchers when studying rice fields, namely: the complex correlation between both copolar channels (in amplitude and phase), entropy and alpha angle obtained by the eigenvalue/vector decomposition of the $2 \times 2$ coherency matrix, and the outputs of a model-based decomposition of the same matrix. Consequently, an effort is made to explain the influence of incidence angle (i.e. observation geometry) upon them.

\section{TEST SITE AND DATA SETS}

The test site consists of an area of $30 \mathrm{~km}$ x $30 \mathrm{~km}$ close to the mouth of the Guadalquivir river, Sevilla, Spain $\left(37.15^{\circ} \mathrm{N}, 6.10^{\circ} \mathrm{W}\right)$, where rice is cultivated annually from May to October. General rice species in this area is Oryza sativa L.. The specific variety cultivated in the monitored fields corresponds to a long grain type named puntal, quite common in Spain and other similar temperate regions.

During 2009 all the fields of the whole site exhibited a quite uniform development, thanks to the good availability of water and to the integrated production scheme implemented in this site. In fact, all parcels yielded productions between 8.5 and 10.5 tons/ha, being one of the most productive sites in the world. Six specific parcels, spread over the whole site, were selected for intensive sampling, with ground measurements on a weekly basis. The weekly measurements include phenological stage and total vegetation height. Phenology is expressed in the general BBCH scale for cereals [13]. Additional details of the campaign can be found in [6], [7]. Farming practices in this area ensure that the ground is always flooded during the cultivation period.

Three parallel time series of HHVV dual-pol images were set up, each one obtained with a different incidence angle, namely 22, 30 and $40 \mathrm{deg}$. The acquisitions with 22 and $40 \mathrm{deg}$. correspond to descending orbits, acquired at 6:30 a.m. The images at $30 \mathrm{deg}$. were acquired with ascending orbits at 6:15 p.m. The final schedule of the images actually acquired is illustrated in Fig. 1 together with the evolution of phenology of the monitored parcels. There are 14 images with 22 deg., 15 with 30 deg., and only 8 with 40 deg. The missing images (all at $40 \mathrm{deg}$. incidence) are due to conflicts with other users' orders or with maintenance tasks of the sensor. In the best cases, when all passes per cycle are effectively acquired, the temporal gaps between acquisitions are only 2, 4 and 5 days, this providing very frequent coverage of the site.

\section{ANALYSIS OF THE INFLUENCE OF INCIDENCE ANGLE UPON DIFFERENT POLARIMETRIC OBSERVABLES}

The copolar polarimetric response of these rice fields at X-band and $30 \mathrm{deg}$. incidence angle is thoroughly described in [7]. Therefore, this section will concentrate on the differences and common aspects found when we change the incidence angle to 22 or $40 \mathrm{deg}$. In order to compare the different incidence angles, we will show the evolutions of some observables for a single parcel. In this way the identification of common features and discrepancies between incidence angles is easier than by representing the response from all parcels in the same figures. The comments included in the following are general for all parcels, since all of them present very similar responses. Particular features and specific ranges of values will be pointed out when necessary. Average and standard deviation of all observables are computed at parcel level and plotted as a function of phenology. Note that phenology, instead of date, is used for the x-axis to ease the physical interpretation of 


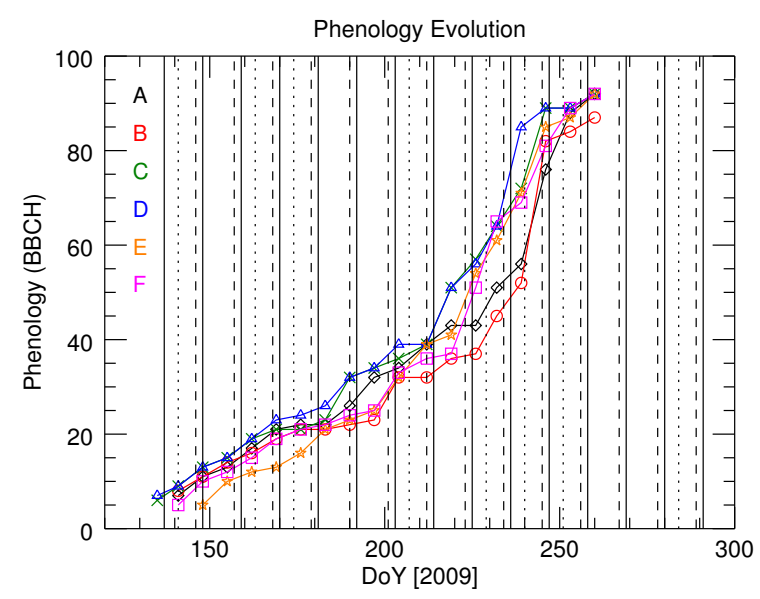

Fig. 1. Evolution of the phenology at the six monitored parcels (labelled from A to F) during the ground campaign in 2009, plotted as a function of day of year (DoY). Vertical black lines denote the dates of acquisition of the TerraSAR-X images: Dashed (- -) for $22 \mathrm{deg}$.; Solid (-) for $30 \mathrm{deg}$; Dotted (..) for $40 \mathrm{deg}$.

the data.

\section{A. Backscattering coefficients}

Figure 2 shows the backscattering coefficient $\sigma_{0}$ at $\mathrm{HH}$ and VV polarisations. Due to the low level of the radar backscatter at all channels during the early stages (stages 0 to 15-20) and at VV during the advanced vegetative phase (stages 30-50), when compared to the noise equivalent sigma zero (NESZ), we employ here the radiometrically enhanced (RE) products [14]. Later in the text we will comment the consequences of getting these echoes around or below the noise level on different observables.

The dynamics of both $\mathrm{HH}$ and VV are quite similar for all incidence angles. In all cases there is low backscatter at the initial stages (which correspond to a flooded ground) and a sharp peak around stage 20 as a consequence of the emergence of the plants and the initial tillering. Then, there is a decrease in both $\sigma_{0}$ (more pronounced in $\mathrm{VV}$ than in $\mathrm{HH}$ ) and a convergence to a value around $-10 \mathrm{~dB}$ at the end of the season (see [7] for a detailed physical explanation). Nonetheless, some effects are more evident at some incidence angles, and the exact values of backscatter at each angle are different in many cases. This aspect is described next.

At the beginning of the cultivation cycle, when the radar echo comes from the water surface, $\sigma_{0}$ depends on the surface roughness, which is induced by wind and, therefore, may vary importantly between acquisitions. Under the same conditions, surface backscatter would be higher at steep incidence angles (22 deg.) than at shallow ones (40 deg.), as it is observed for this parcel. However, the level at the intermediate angle (30 deg.) is the highest one, which can be explained only on the basis of the changes in wind conditions during acquisitions. It turns out that the time, for each day, at which the maximum wind speed was registered in this site during 2009 is predominantly from 2 p.m. to 6 p.m., so we should expect stronger winds at 6:15 p.m. (time of the 30-degree acquisitions) than at 6:30 a.m (time of the other two acquisitions). The maximum wind speed varies from 3 to $13 \mathrm{~km} / \mathrm{h}$, depending on the day, and its direction is predominantly from SW. The test site is on an extremely flat area, located at $30 \mathrm{~km}$ from the sea coast, so the wind conditions are very homogeneous over the whole site. Consequently, the wind-induced roughness, which is always variable in time and space, is considered here to be driven mostly by time. This justifies the observed higher radar backscatter at $30 \mathrm{deg}$.

It is important to highlight that all angles are sensitive enough to the emergence of plants. In principle the more oblique the incidence, the better should be the detection of the initial plant stems emerging from the water surface [6], and especially at vertical polarisation, since the interaction of the vertical elements of the plants with the incoming waves is polarisation dependent. However, the timing of the acquisitions is key to appreciate the response of the initial stems. In our case, the time series gathered at $40 \mathrm{deg}$. does not provide 

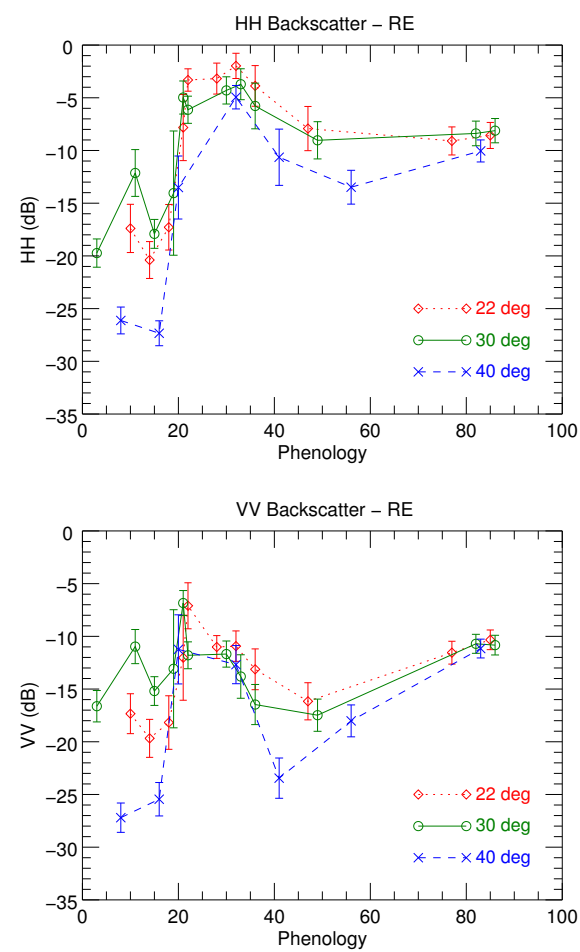

Fig. 2. Evolution of the backscattering coefficients at all incidence angles for one parcel as a function of phenology. Top: HH, Bottom: VV

required time sampling, since there are missing images. Therefore, it depends on the particular case of each parcel whether it can be observed or not.

During the advanced vegetative and early reproductive stages (20-60) we can observe that $\sigma_{0}$ at $\mathrm{HH}$ channel is ordered from steeper angles (higher levels) to shallower angles (lower levels), the maximum difference being about $4 \mathrm{~dB}$. At vertical polarisation the effect is more pronounced, especially around stage 40 , when the VV backscatter at $40 \mathrm{deg}$. falls more than $5 \mathrm{~dB}$ below the value at $30 \mathrm{deg}$. In general, we can state that the dynamic range of the whole evolution at both $\mathrm{HH}$ and VV channels is larger for shallower angles than for steeper angles. Since the backscatter from the bottom of the rice plants is dominated by the double bounce between stems and the water surface (direct surface backscatter is negligible when plants are developed), which at $\mathrm{HH}$ is almost independent from incidence angle, we can state that the influence of incidence angle is due to the extinction inside the vegetation volume. The total attenuation depends on the length of the path followed by the waves, which is larger for shallower angles, and hence the lower absolute values at $40 \mathrm{deg}$.

It is also interesting to see that the backscatter at all angles, and for both polarisations, is quite similar at the final stages (75-90). The independence from incidence angle confirms the physical interpretation provided in [7], where the rice fields at these stages were identified as random volumes. Since the scattering from a random volume should not depend on incidence angle, the angular diversity of these observations provides the same conclusion of previous works, which were based only on polarimetric indicators (e.g. high entropy). Note that the claimed independence from incidence angle of the scattering from a random volume is strictly true only for $\gamma_{0}$, whereas it is not for the backscattering coefficient $\sigma_{0}$. Anyway, for the angular range observed here (from 22 to $40 \mathrm{deg}$.), the influence of incidence angle on $\sigma_{0}$ is less than $1 \mathrm{~dB}$.

These two backscattering coefficients provide a limited observation space, with some ambiguities (two or more situations produce the same values) and a clear influence of incidence angle at some key stages. However, many other observables with clear physical interpretation can be exploited when coherent copolar polarimetry is available, so the next sections explore their evolution along the growth cycle of rice as a function of incidence angle. 


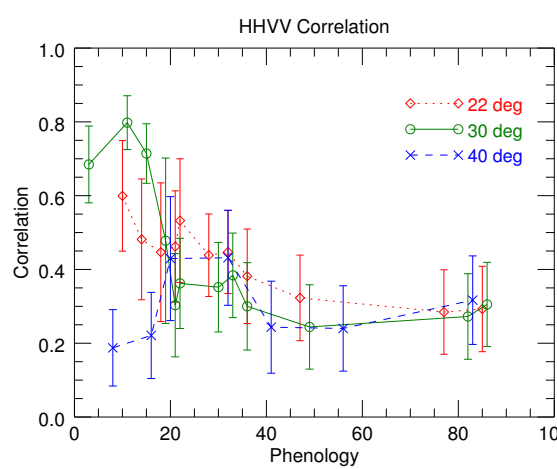

(a)

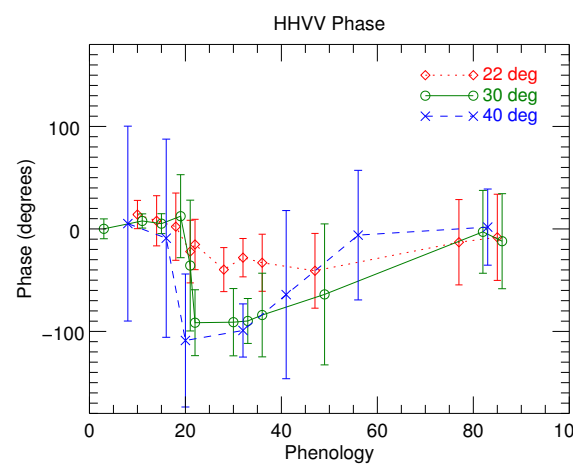

(b)

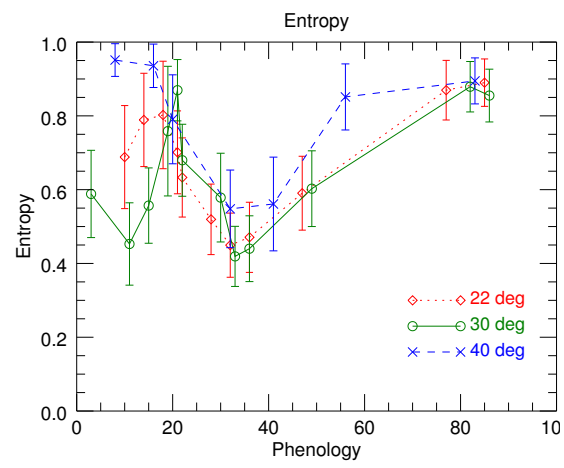

(c)

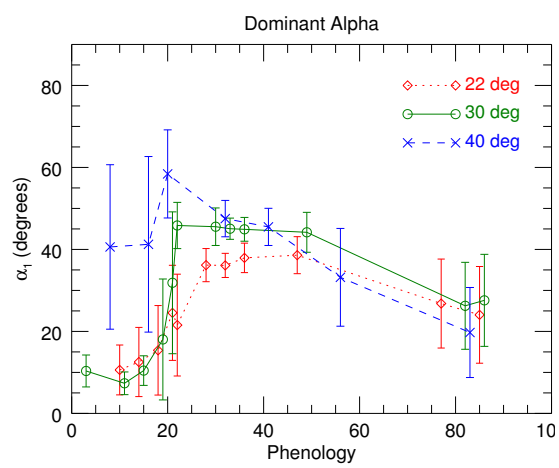

(d)

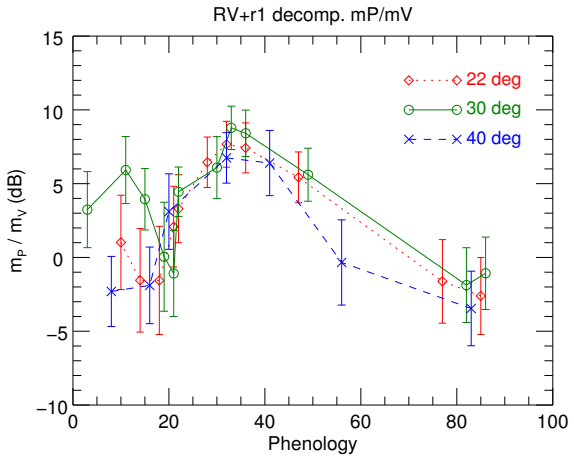

(e)

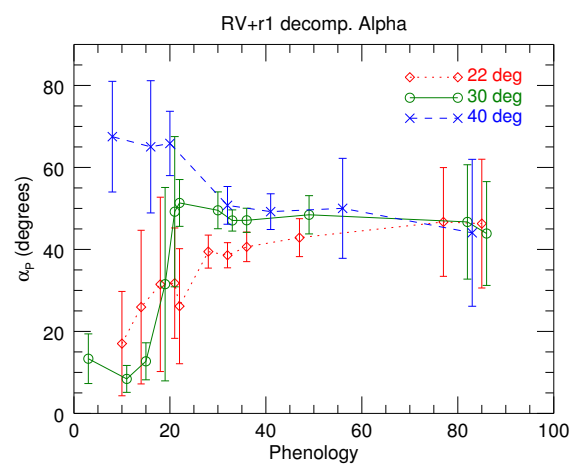

(f)

Fig. 3. Evolution of polarimetric observables at all incidence angles for one parcel as a function of phenology. Left column: Normalised correlation (a) and the phase difference (b) between HH and VV. Central column: Entropy (c) and the dominant alpha angle $\alpha_{1}$ (d). Right column: Outputs of the random volume + rank-1 decomposition: Ratio of the polarised component over the volume component (e), and alpha angle of the polarised component (f).

\section{B. Correlation and phase difference between $H H$ and $V V$}

Correlation between $\mathrm{HH}$ and $\mathrm{VV}$ can be easily interpreted for this scene at three different growth phases (see Fig. 3a). First, during the early stages (0-20) the backscatter from the water surface imposes a high correlation. However, for this parcel this is noticed at the 30-degree incidence, but not at the other two incidences. The reason of the lower coherence at 22 , and especially at $40 \mathrm{deg}$., is the low backscatter with respect to NESZ, as explained before. Noise decreases the correlation between the two copolar channels. In fact, comparing Figs. 2 and $3 \mathrm{a}$, we can see that the lower the backscatter coefficients, the lower the correlation. Second, between stages 25-30 and 55-60, the extremely low backscatter at VV channel reduces the correlation between both copolar signals. In fact, noise affects this parameter, especially at $40 \mathrm{deg}$. incidence, producing correlations as low as 0.2 . Finally, when plants approach the late stages, characterised by the physical morphology of a random volume, coherence tends to $1 / 3$ at all incidences, as expected from theory.

The phase difference between $\mathrm{HH}$ and VV presents a common behaviour for all three incidences (see Fig. 3b), coincident with the explanation shown in [7] for $30 \mathrm{deg}$. However, the peak value at the plants emergence and initial tillering depends on the incidence angle. The largest absolute value is obtained for the shallowest incidence ( $40 \mathrm{deg}$.), reaching values between -90 and -130 deg., depending on the parcel. For the 30 -deg. incidence, the extreme values are in a range between -80 and -120 deg., hence very similar to the $40 \mathrm{deg}$. values. However, at the steepest incidence ( $22 \mathrm{deg}$.$) , the peak phase difference only reaches values$ down to $-50 \mathrm{deg}$., not being below $-25 \mathrm{deg}$. for some parcels. Therefore, at $22 \mathrm{deg}$. there is a worse sensitivity to this phenological stage than at 30 and $40 \mathrm{deg}$.

It is also important to note that at $40 \mathrm{deg}$. the extreme phase difference is reached at earlier stages (even at 15). The stages for the peak values move to $17-22$ at $30 \mathrm{deg}$., and to 20-25 at $22 \mathrm{deg}$. Consequently, not only is the peak value less pronounced at $22 \mathrm{deg}$., but it appears at stages where plants are more developed. 
From the monitoring viewpoint, it would be more complicated to use the copolar phase difference at $22 \mathrm{deg}$. than at 30 and $40 \mathrm{deg}$. to detect the emergence of plants. The acquisition geometry modifies the sensitivity to the presence of the first tillers, being more sensitive at shallower incidences. A similar effect was noticed at C-band in [15] with respect to X-band, due to the size of the plants elements in terms of the wavelength.

\section{Entropy and dominant alpha angle}

We take a look in this section at two observables defined specifically for dual-pol data in [16]: entropy $H$ and dominant scattering mechanism, $\alpha_{1}$ (see Fig. 3c and 3d). The radar response of rice fields at X-band is characterised by two stages with low entropy: the early dates, when the only contribution to backscatter is a low surface component (there is no other element above water in the fields), and the advanced vegetative phase (stages 25-50) when plants are fully developed and then backscattering is linearly polarised due to the extreme attenuation suffered by the VV channel. The second of these stages is easily identified at all incidence angles, with entropy values below 0.65 . However, at the initial dates there are high entropies at $22 \mathrm{deg}$. incidence and, especially, at $40 \mathrm{deg}$. The cause of such misbehaviour is, in accordance with previous comments for the coherence between $\mathrm{HH}$ and $\mathrm{VV}$, the low backscatter level, which makes entropy to be increased by noise.

There are two stages for which entropy is high: around the start of tillering (stage 20) and at maturation (stages 70+). The second case produces high entropy due to the random volume nature of the scene, whereas the first one is due to the simultaneous presence of two scattering mechanisms (none of them dominating), which are surface backscatter from the water and double-bounce backscatter from the stems-water interaction (see [7] for a detailed explanation). Both high entropy stages are easily recognised at 30 and 40 deg., for all parcels, reaching values around 0.9 , but not the first of them at $22 \mathrm{deg}$., since entropy does not go beyond 0.8 for any parcel around stage 20. This is in agreement with previous comments: the steepest incidence is less sensitive to the presence of the first tillers (scattering is still dominated by the surface contribution) and, on the contrary, all incidences respond equally to the random volume at the end of the season.

Following the same discussion, the dominant alpha angle $\alpha_{1}$ provides information about which scattering type dominates at each stage, being meaningful whenever entropy is not high. This is the case of the initial stages, with $\alpha_{1}$ close to 0 meaning surface scattering, and during the advance vegetative phase, since $\alpha_{1} \approx$ $45 \mathrm{deg}$. means linearly polarised backscatter. Once again, at $40 \mathrm{deg}$. we cannot see low values of $\alpha_{1}$ for this parcel during the early dates because of the extremely low backscatter level and, hence, the high entropy. More importantly, we observe that $\alpha_{1}$ does not reach $45 \mathrm{deg}$. at stages $25-50$ for the $22 \mathrm{deg}$. incidence angle, contrarily to the values observed for the other two incidences. In this case, and consistently for all parcels, the value of $\alpha_{1}$ during the advanced vegetative phase is around $35 \mathrm{deg}$. This can be explained physically by the smaller effect of extinction at steeper incidences, which makes the VV backscatter, with respect to $\mathrm{HH}$, not as low as for more oblique incidences.

During the plants emergence and initial tillering (around stage 20), we also observe an important effect of the incidence angle on $\alpha_{1}$, which is present in all parcels. At 40 deg. incidence, this parameter reaches $60 \mathrm{deg}$. or more for all parcels, which means that the double-bounce contribution coming from the interaction between water surface and stems dominates the backscatter. At $30 \mathrm{deg}$. incidence, the peak value is between 45 and 60 deg., depending on the parcel, which, in comparison with the $40 \mathrm{deg}$. incidence, shows a lower sensitivity to the presence of the initial tillers. Finally, at $22 \mathrm{deg}$. incidence, $\alpha_{1}$ at this stage ranges from 25 to $40 \mathrm{deg}$., hence being in many cases even lower than the value measured during the advanced vegetative phase. Once again, the steepest incidence is not as sensitive during this stage to the development of the plants. Therefore, accordingly with the comments for the phase difference between HH and VV (Section III-B), the transition observed in this observable is sharper and more pronounced for more oblique incidences, which should be taken into account carefully when devising a monitoring strategy based on combining different angles. 


\section{Model-based decomposition}

The outputs of a model-based decomposition proposed in [7] are studied here. With this approach, the rice response is decomposed into two terms: backscatter coming from a random volume, $m_{V}$, and backscatter coming from a remaining polarised term, $m_{P}$, characterised by a rank- 1 coherency matrix. The observables shown in Fig. $3 \mathrm{e}$ and $3 \mathrm{f}$ are the ratio of the amount of both terms, $m_{P} / m_{V}$, and the alpha angle of the polarised component, $\alpha_{P}$, which indicates its type of scattering mechanism.

During the early stages, the polarised component should dominate the response at all incidences, since there is no vegetation producing any volume scattering. However, this is only the case at $30 \mathrm{deg}$. incidence due to the low backscatter, with respect to NESZ, found at 22 and $40 \mathrm{deg}$. In such circumstances, noise is misinterpreted as volume scattering. Then, at plant emergence and initial tillering (stage 20) there is a jump in both components, $m_{V}$ and $m_{P}$, for all incidence angles. The volume component decreases some dB just after that peak at stage 20, whereas the polarised component maintains its level up to stage 30-35 and then decreases, and hence the observed variation in their ratio. The $m_{P} / m_{V}$ ratio exhibits a common behaviour for all parcels and incidence angles, with a maximum value of 5 to $8 \mathrm{~dB}$ at stage 30, approximately, and a continuous decrease towards slightly negative values $(-5$ to $-1 \mathrm{~dB})$ at stage $70-80$ and constant afterwards. Therefore, this observable shows perfectly the change in the scattering properties of the scene from stage $20-25$ to the end.

Finally, the evolution of $\alpha_{P}$ is quite similar to $\alpha_{1}$ up to stage 50. From that stage onwards, however, $\alpha_{1}$ moved to low values (below 40 deg.) and with different final values as a function of incidence angle. In contrast, $\alpha_{P}$ tends to $45 \mathrm{deg}$. from stage 50 to the end of the season. This happens for all parcels at all incidence angles. As explained in [7], these $\alpha$ angles are retrieved in a different way, hence their different values at these late stages. With the model-based decomposition, we firstly estimate and extract the volume component (originated at the vegetation random volume), so it is easier to identify the remaining term as the underlying polarised contribution. In the eigendecomposition, we do not make such a separation of the volume term before estimating the dominant scattering mechanism.

\section{SUMmARY AND CONCLUSIONS}

The influence of incidence angle upon X-band polarimetric observables derived from coherent copolar measurements over rice fields has been analysed in this letter. Despite the limited amount of available data, this study provides guidelines about what to expect when using X-band multi-angular data in similar situations. We have shown that some stages in the growth cycle exhibit a consistent radar response at all incidence angles, whereas others behave differently depending on the observation geometry. Specifically, the polarimetric observables related with dominance of scattering mechanisms (e.g. entropy and ratio of volumeto-polarised components) and with the type of dominant scattering mechanism (alpha angles) do not vary significantly with angle during the early and advanced vegetative stages and at the maturation phase, so they could be exploited in the future for monitoring purposes combining different beams and orbits. However, observation geometry influences the sensitivity to the presence of the first stems and tillers, since they are more easily detected at shallower incidences than at steeper ones. In previous works with a single incidence angle we proposed a combination of 3 parameters for rice monitoring: dominant alpha, entropy and span (only for low backscatter areas). These three seem to be the most suitable for further studies since they exhibit better angle invariance than backscatter alone.

\section{ACKNOWLEDGEMENTS}

The authors would like to thank the support of M. Cano, F. Carrascal and S. Aparicio (Federacion de Arroceros de Sevilla) for providing the ground measurement data and for their helpful comments. All SAR images have been provided by the German Aerospace Centre (DLR) in the framework of projects LAN0021 and LAN0234 of the pre-launch AO of TerraSAR-X. 


\section{REFERENCES}

[1] T. Kurosu, M. Fujita, and K. Chiba, "Monitoring of rice crop growth from space using the ERS-1 C-band SAR," IEEE Trans. Geosci. Remote Sensing, vol. 33, no. 4, pp. 1092-1096, July 1995.

[2] T. Le Toan et al., "Rice crop mapping and monitoring using ERS-1 data based on experiment and modeling results," IEEE Trans. Geosci. Remote Sensing, vol. 35, no. 1, pp. 41-56, Jan. 1997.

[3] J.-Y. Koay et al., "Paddy fields as electrically dense media: Theoretical modeling and measurement comparisons," IEEE Trans. Geosci. Remote Sensing, vol. 45, no. 9, pp. 2837-2849, Sept. 2007.

[4] A. Rosenqvist, "Temporal and spatial characteristics of irrigated rice in JERS-1 L-band SAR," Int. J. Remote Sensing, vol. 20, pp. 1567$1587,1999$.

[5] A. Bouvet, T. Le Toan, and N. Lam-Dao, "Monitoring of the rice cropping system in the Mekong delta using ENVISAT/ASAR dual polarization data," IEEE Trans. Geosci. Remote Sensing, vol. 47, no. 2, pp. 517-526, Feb. 2009.

[6] J. M. Lopez-Sanchez, J. D. Ballester-Berman, and I. Hajnsek, "First results of rice monitoring practices in Spain by means of time series of TerraSAR-X dual-pol images," IEEE J. Selected Topics in Applied Earth Observations and Remote Sensing, vol. 4, no. 2, pp. 412-422, June 2011.

[7] J. M. Lopez-Sanchez, S. R. Cloude, and J. D. Ballester-Berman, "Rice phenology monitoring by means of SAR polarimetry at X-band," IEEE Trans. Geosci. Remote Sensing, vol. 50, no. 7, pp. 2695-2709, July 2012.

[8] Y. Inoue and E. Sakaiya, "Relationship between X-band backscattering coefficients from high-resolution satellite SAR and biophysical variables in paddy rice," Remote Sensing Letters, vol. 4, pp. 288-295, Mar. 2013.

[9] B. A. M. Bouman et al., ORYZA2000: A crop growth simulation model for rice, IRRI and Wageningen University, https://sites.google.com/a/irri.org/oryza2000.

[10] F. Holecz et al., "An operational remote sensing based service for rice production estimation at national scale," in Proceedings of the ESA Living Planet Symposium, SP-722, Edinburgh, U.K., Sept. 2013.

[11] Rice Knowledge Bank, IRRI, http://www.knowledgebank.irri.org/rice.htm.

[12] Y. Inoue et al., "Season-long daily measurements of multifrequency (Ka, Ku, X, C, and L) and full-polarization backscatter signatures over paddy rice field and their relationship with biological variables," Remote Sensing of Environment, vol. 81, pp. 194-204, 2002.

[13] U. Meier, Ed., Growth Stages of Mono- and Dicotyledonous Plants. BBCH Monograph, 2001.

[14] T. Fritz, "Tips \& tricks for spectral, radiometric and geometric interpretation of TerraSAR-X products. Part I," in Proceedings of the 3rd TerraSAR-X Science Team Meeting, Wessling, Germany, 2008.

[15] J. M. Lopez-Sanchez, F. Vicente-Guijalba, J. D. Ballester-Berman, and S. R. Cloude, "Polarimetric response of rice fields at C-band: Analysis and phenology retrieval," IEEE Trans. Geosci. Remote Sensing, vol. 52, no. 5, pp. 2977-2993, May 2014.

[16] S. R. Cloude, "The dual polarization entropy/alpha decomposition: a PALSAR case study," in Proceedings of the 3rd PolInSAR Workshop, Frascati, Italy, Jan. 2007. 